\title{
Guest editorial for special issue on biometric systems and applications
}

\author{
Muhammad Khurram Khan
}

Published online: 26 May 2010

(C) Springer Science+Business Media, LLC 2010

Biometrics is an automated method of recognizing a person based on her physiological or behavioral characteristics, and is potentially a powerful and reliable method for personal identification. Biometrics is a multidisciplinary research area of different subjects e.g. signal processing, pattern recognition, computer vision, biology, and statistics. Due to their highly secure and trustworthy characteristics, biometrics technologies are becoming the foundation of an extensive array of highly secure identification and personal verification solutions. In our daily life, we observe many applications of biometrics around us e.g. border control, driver license, time management and attendance, authentication at security gate, and surveillance etc.

The significance of biometrics is also underscored by the rapidly growing academic and research activities devoted to this field, and by a large number of annually organized events by different institutions. However, the key challenge to the research community is to propose and develop integrated solutions that deal with the entire gamut of research problems from sensors and data acquisition to biometric data analysis, systems design and real applications.

This special issue is a collection of original papers that cover a wide range of topics from design, implementation, security, and application of biometrics systems. As a whole, this special issue contains a diverse collection of high-quality papers authored by eminent researchers in the field. There were total 24 submissions from several countries around the world and through a rigorous peer-review process, only 8 submissions got final acceptance for the publication.

M.K. Khan $(\varangle)$

Center of Excellence in Information Assurance (CoEIA),

King Saud University, Riyadh, Kingdom of Saudi Arabia

e-mail: mkhurram@ksu.edu.sa
The first paper by B. Tunc and M. Gokmen presents a manifold learning-based face recognition system under illumination variations. They develop an approach based on nonlinear manifold embedding to define a linear subspace for illumination variations. Their framework utilizes an optimization scheme to calculate the bases of the subspace. Since the optimization problem does not rely on the physical properties of the factor, their framework can also be used for other types of factors such as pose and expression variations.

The second paper by Choi et al. proposes an incremental face annotation framework for sharing and publishing face images under a large-scale web platform such as a social network service. The main contribution of their work is a computationally efficient incremental face recognition method in terms of time and memory allocation for web platforms where both training samples and users grow over time.

The next paper by Pan et al. deals with the non-intrusive liveness detection mechanism of face recognition against spoofing. They employ combination of the clues of eyeblinks and scene context for liveness detection of facial images in front of camera. The scene context clue is to detect video imposters that the eyeblink clue cannot detect and the eyeblink clue is to detect trimmed photograph of imposters that the scene context clue cannot detect.

The fourth paper by Khan et al. presents a privacypreserving and tokenless chaotic facehashing scheme to overcome the irrevocability problem of face-biometric. In their scheme, users don't need to posses USB tokens in generating pseudorandom sequences, which is a cost-effective solution. Besides, their scheme minimizes the system complexity with simple operations to attain secure and revocable FaceHash.

The paper by H. Beigi and J.A. Markowitz presents a proposal for speaker recognition which they call Standard $\mathrm{Au}-$ dio Format Encapsulation (SAFE). It supports all types of 
audio interchange operations while, at the same time, limiting the audio formats to a small set of widely-used, open standards. The SAFE proposal has been incorporated into speaker-recognition data interchange draft standards by different standardization agencies of biometrics.

The next paper by J. Galbally et al. performs evaluation of direct attacks on fingerprint verification systems. They used minutiae-based and ridge feature-based fingerprint recognition systems to perform experimentations on real and fake fingerprint images. They proposed that liveness detection procedures or multimodal authentication are two possible countermeasures against this type of fraudulent actions in biometric systems.

The paper by Lee et al. presents a non-invertible revocable bits extraction technique by means of quantizing the facial data from two feature extractors in the phase domain, which they called aligned feature-level fusion phase quantization (AFPQ). In this technique, Lee et al. utilize helper data to achieve the revocability requirement of bits extraction. The feature averaging and remainder normalization technique are integrated with the helper data to reduce feature variance within the same individual and increase the distinctiveness of bit strings of different individuals to achieve good recognition performance.

The last paper by Badrinath et al. proposes a palmprint based verification system which uses low-order Zernike moments of palmprint sub-images. They used Euclidean distance to match the Zernike moments of corresponding subimages of query and enrolled palmprints. These matching scores of sub-images are fused using a weighted fusion strategy. Their system can also classify the sub-image of palmprint into non-occluded or occluded region and verify user with the help of non-occluded regions.

Acknowledgements I, being the Guest Editor, would like to express sincere thanks to all the people who have contributed their time and efforts in making this special issue a success. I thank all the authors who contributed their papers for this special issue. I am also full of gratitude to the reviewers who scrutinized the submitted manuscripts and recommended modifications and revisions in enhancing the quality of the accepted papers. In addition, I am also grateful to Mr. Jackie James for his assistance. Last, but certainly not the least, I would like to pay my special thanks to the Editor-in-Chief, Prof. Bezalel Gavish, for his encouragement and strong support during the preparation of this special issue. 